\title{
Systemic lupus erythematosus
} report and literature review

\author{
Junxian Wen ${ }^{1,2+}$, Weijie Chen ${ }^{2 \dagger}$, Lu GaO $^{3}$, Xiaoyuan Qiu ${ }^{2}$ and Guole Lin ${ }^{2 *}$ (D)
}

\begin{abstract}
Background: Intestinal pseudo-obstruction (IPO) accompanied by hepatobiliary dilatation and ureterohydronephrosis is extremely rare in systemic lupus erythematosus (SLE). This triad is also called visceral muscle dysmotility syndrome (VMDS). Only 9 cases have been reported in the literature. Here, we report a rare case of VMDS with mechanical intestinal obstruction that was clinically relieved by surgery.

Case presentation: This report refers to a 31-year-old woman with SLE and gastrointestinal symptoms presented as abdominal pain, nausea and stoppage of the passage of flatus or stool without obvious reasons. The patient suffered from severe abdominal distension because of massive flatulence. Contrast-enhanced computed tomography (CT) of the abdomen performed in our hospital showed localized stenosis of the bowel, ureterohydronephrosis, and biliary tract dilatation. Endoscopy showed a stenotic segment located in the sigmoid colon. The colon biopsy samples suggested that the stenosis was caused by inflammatory tissues. Biochemical investigations showed hypoalbuminemia, electrolyte disturbance and decreased C3. Antinuclear antibody was positive. After careful assessment, transverse colostomy was performed for this patient. Gastrointestinal symptoms were clinically relieved after the surgery.
\end{abstract}

Conclusion: To the best of our knowledge, no VMDS patients have presented with mechanical ileus before. This case is the first documented occurrence of SLE with VMDS and mechanical intestinal obstruction symptoms relieved by surgery. Due to the low incidence of this condition, no standard treatment regimen has been established. However, surgical treatment offers significant benefit in specific situations.

Keywords: Systemic lupus erythematosus, Mechanical intestinal obstruction, Visceral muscle dysmotility, Intestinal pseudo-obstruction, Case report

*Correspondence: guolelin2002@163.com

†Junxian Wen and Weijie Chen have contributed equally to this work and should be considered co-first authors

${ }^{2}$ Department of Surgery, Peking Union Medical College Hospital, Peking Union Medical College, Chinese Academy of Medical Sciences, No. 1 Shuaifuyuan, Dongcheng District, Beijing 100730, People's Republic of China

Full list of author information is available at the end of the article

\section{Background}

Systemic lupus erythematosus (SLE) is a prevalent autoimmune disease which presents with various clinical features and manifestations [1]. Intestinal pseudo-obstruction (IPO) is one of the most uncommon gastrointestinal system symptoms in SLE [2]. In some rare cases, patients could present with IPO and 
pyeloureterectasis and biliary tract dilatation simultaneously. This rare triad is named generalized megaviscera of lupus (GML) or visceral muscle dysmotility syndrome (VMDS) $[3,4]$. To date, only 9 cases have been reported in the literature $[2,3,5,6]$. However, here, we present an even rarer case with VMDS and mechanical intestinal obstruction secondary to SLE. To our knowledge, this is the first report of an SLE patient presenting with these symptoms simultaneously.

\section{Case presentation}

The patient was a 31-year-old woman with a 15-year history of erythema on the cheek, swelling and pain of both knees, and abdominal distension. She had a history of idiopathic thrombocytopenia verified in December 1997. The patient had been maintained on prednisone $10 \mathrm{mg}$ qd. In late March 2021, the patient suddenly stopped defecating, and this was accompanied by abdominal distension, intermittent nausea and retching. An indwelling gastric tube was placed in another hospital and the patient was treated with daily enemas. Gastric tube drainage was $300-400 \mathrm{ml}$ per day. In mid-April, she was prescribed methylprednisolone $500 \mathrm{mg} \times 3 \mathrm{~d}$ shock treatment in addition to cyclophosphamide $0.2 \mathrm{~g}$ iv qod. The abdominal distension was relieved, but there was still no bowel movement. Finally, the patient came to our hospital on May 6 because of paroxysmal colic in the lower abdomen.

On admission, findings from physical examination were as follows: body temperature was $36.6^{\circ} \mathrm{C}$, pulse was $84 / \mathrm{min}$, respiratory rate was $18 / \mathrm{min}$, and blood pressure was $127 / 101 \mathrm{mmHg}$. The patient entered the room in a wheelchair with a gastric tube and a right subclavian central venous catheter. The patient's abdomen was extremely distended, with tenderness in the left lower quadrant (Fig. 1) but no rebound tenderness. The bowel sounds were very weak and hardly audible. Liver and spleen were not palpable in the subcostal and subxiphoid regions.

Blood tests showed positive antinuclear antibody (1:160); anti-dsDNA antibody, (-); antiRNP, (-); anti-Sm, $(-)$; anti-SSA, (-); anti-SSB, (-); anti-ribosomal P, (-); anti-cardiolipin antibody, $(-)$; and lupus anticoagulant, 1.2. In immunoserological testing, CRP was $33.08 \mathrm{mg} / \mathrm{l}$; C3, $0.538 \mathrm{~g} / \mathrm{l}$; and C4, $0.408 \mathrm{~g} / \mathrm{l}$. Biochemical testing showed serum albumin was $27 \mathrm{~g} / \mathrm{l}$; ALT $40 \mathrm{U} / \mathrm{L} ;$ TBil
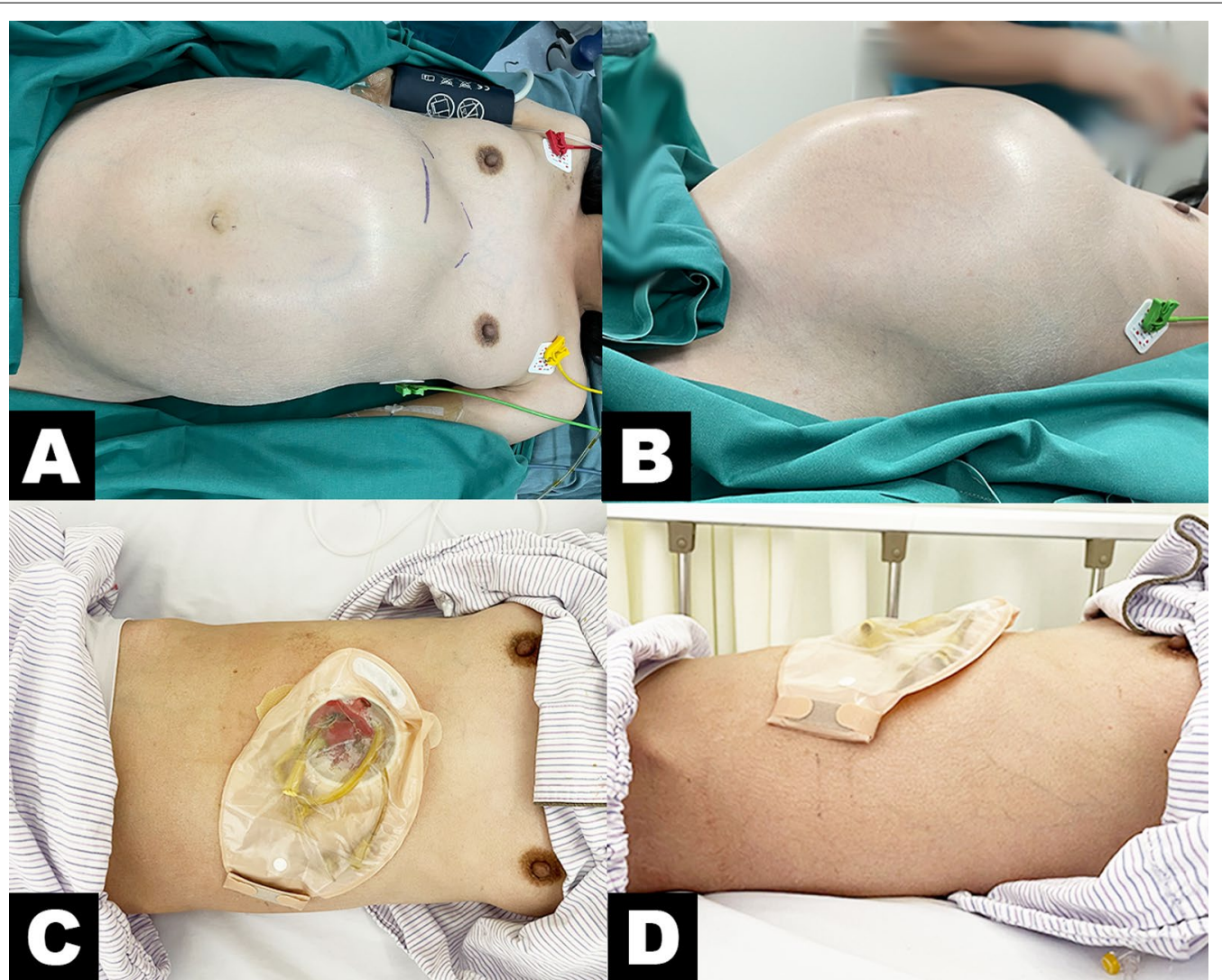

Fig. 1 Preoperative and postoperative images of the abdomen of the patient. Top view (A) and lateral view (B) show patient's extremely distended abdomen before surgery. The patient's abdomen flattened after surgery as seen in top view (C) and lateral view (D) 
$10.7 \mu \mathrm{mol} / \mathrm{L} ;$ DBil 6.6umol/L; Gamma-glutamyltransferase (GGT) $433 \mathrm{U} / \mathrm{L} ; \mathrm{K}, 3.4 \mathrm{mmol} / \mathrm{L} ; \mathrm{Na}, 137 \mathrm{mmol} / \mathrm{L}$; Ca, $2.07 \mathrm{mmol} / \mathrm{L}, \mathrm{Fe} 14 \mu \mathrm{g} / \mathrm{dL} ; \mathrm{Cr} 67 \mu \mathrm{mol} / \mathrm{L}$; and Urea $6.13 \mathrm{mmol} / \mathrm{L}$. Coombs test was positive, and total urine protein was $1.64 \mathrm{~g} / 24 \mathrm{~h}$.

A contrast-enhanced computed tomography (CT) scan (Fig. 2) on May 28 showed general dilated small and large bowel; dilated intrahepatic and extrahepatic bile ducts and dilated pancreatic ducts; significant enlargement of the gallbladder; bilateral dilatation of the renal pelvis, calyces and ureter; and localized stenosis of the sigmoid colon with dilatation of the upper intestinal canal with fluid flattening. Colonoscopy (Fig. 3) revealed a stenotic segment located $17-20 \mathrm{~cm}$ from the anus, with smooth local mucosa and extremely dilated anterior bowel. The pathological results of sigmoid stenosis also showed chronic mucosal inflammation.

Four days later, a catheter was inserted through the patient's anus to relieve intestinal obstruction, but the abdominal distension did not decrease and was accompanied by abdominal pain and dyspnoea. After several multidisciplinary discussions, we performed a transverse colostomy for this patient. We aspirated approximately
$3000 \mathrm{ml}$ of stool contents during the operation. After the operation, the patient's abdomen flattened, and abdominal distension improved significantly (Fig. 1). CT (Fig. 4) on June 7 showed that the patient's bowel dilatation had improved significantly. Gradually, voluntary bowel movements returned and the patient started enteral nutrition. In this case, early vigorous surgical intervention reversed her deteriorating condition and thus yielded a good outcome. A half year later, we performed a follow-up examination of her symptoms of abdominal distention, and we found that her symptoms did not recur. However, this patient strongly refused any further radical surgery. The histopathology of sigmoid stenosis is still unclear.

\section{Discussion and conclusions}

SLE is an autoimmune disease that predominantly affects women and typically has manifestations in multiple organs, including the skin, kidneys, joints, and gastrointestinal system [7]. Immune system activation in SLE is characterized by exaggerated $\mathrm{B}$ cell and $\mathrm{T}$ cell responses and loss of immune tolerance against self-antigens [8]. The prevalence of SLE in adults ranges from 30 to 150 per 100000 , and the incidence ranges from 2.2 to 23.1

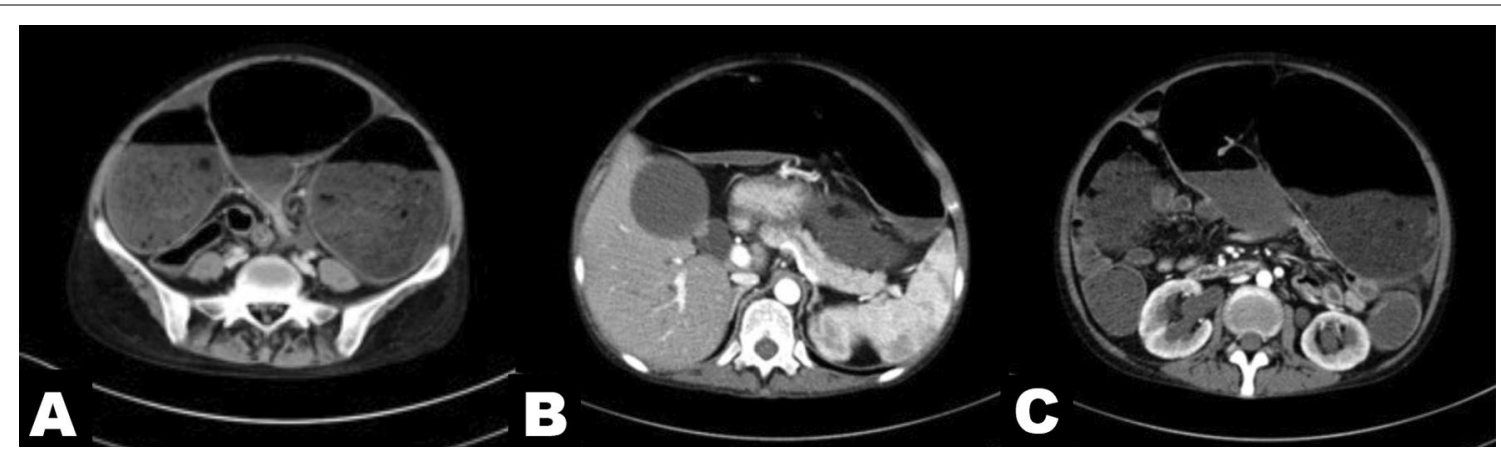

Fig. 2 Computed tomography scans of the abdomen. Localized stenosis of the sigmoid colon and general dilation of the bowel (A); dilated intra- and extra-hepatic bile ducts, dilated pancreatic ducts and enlargement of the gallbladder (B); dilation of the renal pelvis, calyces and ureter (C)

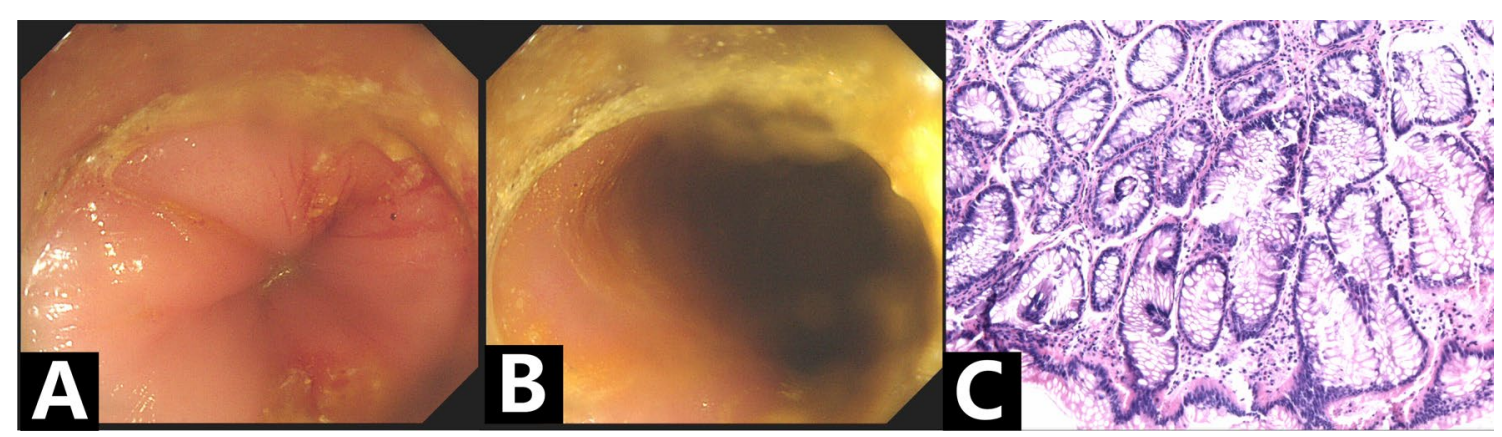

Fig. 3 Colonoscopy and pathology pictures of sigmoid stenosis. Colonoscopy revealed a stenotic segment located $17-20 \mathrm{~cm}$ from the anus (A), with smooth local mucosa and extremely dilated anterior bowel (B). The pathology results of sigmoid stenosis showed chronic mucosal inflammation (C) 


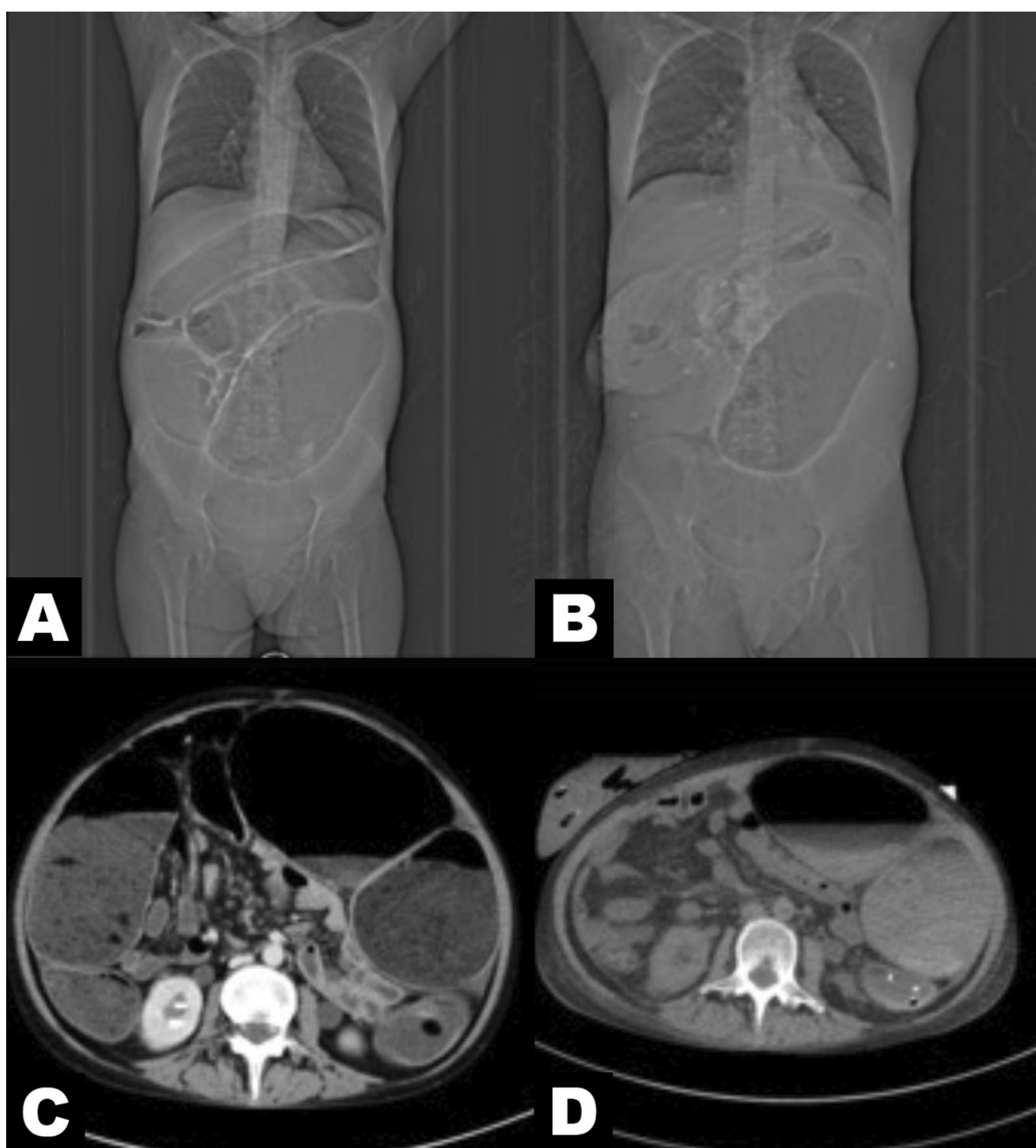

Fig. 4 Preoperative and postoperative abdominal computed tomography scans. Compared to preoperative abdomen reconstruction (A) and axial reconstruction $(\mathbf{C})$, postoperative abdomen reconstruction $(\mathbf{B})$ and axial reconstruction $(\mathbf{D})$ showed that the patient's bowel dilatation had improved significantly after surgery

per 100000 per year [9]. Therapeutic approaches for SLE involve immunomodulation and immunosuppression, which are targeted to reduce disease activity in specific organ manifestations $[1,9]$. However, despite many treatment advances, SLE continues to cause substantial morbidity and mortality.

Our case is an SLE patient who developed severe intestinal complications. IPO is an uncommon complication in SLE and is defined as ineffective intestinal propulsion with the clinical features of intestinal obstruction but without mechanical obstruction. IPO can present as an initial manifestation of SLE. The most common clinical presentation is abdominal pain, followed by nausea and/ or vomiting, abdominal distension, diarrhoea and constipation. This case presented all these symptoms. Pardos-Gea et al. reported in 2005 that IPO was associated with pyeloureterectasis and biliary tract dilatation [6]. Frederick- $D$ et al. and Chen et al. separately named this rare triad generalized megaviscera of lupus (GML) [5] and visceral muscle dysmotility syndrome (VMDS) [3]. According to a previous report, the incidence of VMDS in patients with SLE was less than $0.15 \%$ [2]. The underlying mechanism of VMDS might be vasculitis of the visceral smooth muscles, which leads to muscular damage 
and hypomotility and eventually to concurrent pyeloureterectasis or megacholedochus [10]. Many studies have shown that it can be associated with increased morbidity and mortality [11]. Early recognition of this life-threatening problem and the vigorous use of immunosuppressives can avoid unnecessary ineffective interventions and potentially fatal complications $[3,6]$.

In addition, this patient concurrently suffered from mechanical ileus. To our knowledge, this was never reported before. Because of the presence of sigmoid stenosis, the patient had to undergo emergency surgery. Unfortunately, although we collected tissue from the stenosis during colonoscopy and performed pathological examination, we did not find the exact cause of the stenosis. We speculate that ileus was caused by dysfunction of the visceral autonomic nervous system, which was reported in some cases $[12,13]$. Because enteric neurons are essential for propulsive intestinal motility, the absence of intrinsic enteric neurons could lead to a lack of propulsive motility patterns in the distal bowel. Moreover, inhibitory enteric motor neurons mediate the relaxation of the gut wall during propulsive motility, therefore, an absence of intrinsic inhibitory neurons may also contribute to the persistent constriction of the corresponding segment [14]. We posit that dysfunction of the inhibitory neuron led to localized spastic narrowing of the patient's sigmoid colon and resulted in dilatation of the anterior segment, as in the clinical presentation of Hirschsprung disease [15]. Unfortunately, because of the high intraabdominal pressure and poor health of this patient, we did not remove her diseased bowel in the initial surgery, but only performed a colostomy, and our suspicions were not confirmed.

High-dose steroids and immunosuppressants are the first choice in patients with VMDS secondary to SLE. Early and vigorous immunosuppressive treatment will prevent unnecessary exploratory laparotomy and complications, while dose tapering should be slowed to avoid recurrence3. However, if the patient has a concurrent local intestinal obstruction, autonomic neuropathy should be considered. They should be identified early and the affected segment of the rectum and colon should be removed, or initial primary colostomy should be performed in cases of major health problems. In addition, enteric neurons could potentially be future therapeutic targets for SLE.

In conclusion, if general visceral muscle dysmotility is detected in a patient with SLE and the radiological examination suggests partial intestinal obstruction, doctors should consider the possibility that both VMDS and mechanical intestinal obstruction are present simultaneously. Although more evidence is required to determine the optimal treatment choice, resection of the obstructed bowel or colostomy as an initial treatment is recommended.

\section{Abbreviations}

IPO: Intestinal pseudo-obstruction; SLE: Systemic lupus erythematosus; VMDS: Visceral muscle dysmotility syndrome; CT: Computed tomography.

\section{Acknowledgements \\ Not applicable.}

\section{Authors' contributions}

WJX and CWJ drafted the manuscript, and GL and QXY collected patient data. LGL reviewed and edited the manuscript. All authors have read and approved the manuscript.

\section{Funding}

This study was funded by the Beijing Major Science and Technology Projects (No. D171100002617003). There was no other grant from funding agencies in the public, commercial, or not-for-profit sectors. The funding body had no role in the design of the study and collection, analysis and interpretation of data, or in writing the manuscript.

Availability of data and materials

All information about the patient came from the Department of Rheumatology and Department of Surgery, Peking Union Medical College Hospital.

\section{Declarations}

Ethics approval and consent to participate Not applicable.

\section{Consent for publication}

Written informed consent was obtained from the patient for publication of this case report and any accompanying images. A copy of the written consent is available for review by the Editor-in-Chief of this journal.

\section{Competing interests}

The authors declare that they have no competing interests.

\section{Author details}

${ }^{1}$ Department of Neurosurgery, Peking Union Medical College Hospital, Peking Union Medical College, Chinese Academy of Medical Sciences, No. 1 Shuaifuyuan, Dongcheng District, Beijing 100730, People's Republic of China. ${ }^{2}$ Department of Surgery, Peking Union Medical College Hospital, Peking Union Medical College, Chinese Academy of Medical Sciences, No. 1 Shuaifuyuan, Dongcheng District, Beijing 100730, People's Republic of China. ${ }^{3}$ Department of Breast Surgery, Peking Union Medical College Hospital, Peking Union Medical College, Chinese Academy of Medical Sciences, No. 1 Shuaifuyuan, Dongcheng District, Beijing 100730, People's Republic of China.

Received: 13 July 2021 Accepted: 13 January 2022

Published online: 25 January 2022

\section{References}

1. Fava A, Petri M. Systemic lupus erythematosus: diagnosis and clinical management. J Autoimmun. 2019;96:1-13.

2. Zhang $L, X u D$, Yang $H$, et al. Clinical features, morbidity, and risk factors of intestinal pseudo-obstruction in systemic lupus erythematosus: a retrospective case-control study. J Rheumatol. 2016;43:559-64.

3. Chen $Y Q$, Xue Q, Wang NS. Visceral muscle dysmotility syndrome in systemic lupus erythematosus: case report and review of the literature. Rheumatol Int. 2012;32:1701-3.

4. Kansal A, Jain A, Thenozhi S, Agarwal V. Intestinal pseudo-obstruction associated with biliary tract dilatation in a patient with systemic lupus erythematosus. Lupus. 2013;22:87-91. 
5. Park FD, Lee JK, Madduri GD, Ghosh P. Generalized megaviscera of lupus: refractory intestinal pseudo-obstruction, ureterohydronephrosis and megacholedochus. World J Gastroenterol. 2009;15:3555-9.

6. Pardos-Gea J, Ordi-Ros J, Selva A, Perez-Lopez J, Balada E, Vilardell M. Chronic intestinal pseudo-obstruction associated with biliary tract dilatation in a patient with systemic lupus erythematosus. Lupus. 2005;14:328-30.

7. Dörner T, Furie R. Novel paradigms in systemic lupus erythematosus. Lancet. 2019:393:2344-58.

8. Tsokos GC. Systemic lupus erythematosus. N Engl J Med. 2011:365:2110-21.

9. Durcan L, O'Dwyer T, Petri M. Management strategies and future directions for systemic lupus erythematosus in adults. Lancet. 2019;393:2332-43.

10. Ceccato F, Salas A, Góngora V, et al. Chronic intestinal pseudo-obstruction in patients with systemic lupus erythematosus: report of four cases. Clin Rheumatol. 2008;27:399-402.

11. Khairullah S, Jasmin R, Yahya F, Cheah TE, Ng CT, Sockalingam S. Chronic intestinal pseudo-obstruction: a rare first manifestation of systemic lupus erythematosus. Lupus. 2013:22:957-60.

12. Yukawa S, Tahara K, Shoji A, Hayashi H, Tsuboi N. Acute pan-dysautonomia as well as central nervous system involvement and peripheral neuropathies in a patient with systemic lupus erythematosus. Mod Rheumatol. 2008;18:516-21.

13. Omdal R, Jorde R, Mellgren SI, Husby G. Autonomic function in systemic lupus erythematosus. Lupus. 1994;3:413-7.

14. Kubota M, Suita S, Kamimura T, Ito Y, Szurszewski JH. Electrophysiological properties of the aganglionic segment in Hirschsprung's disease. Surgery. 2002;131:S288-293

15. McKeown SJ, Stamp L, Hao MM, Young HM. Hirschsprung disease: a developmental disorder of the enteric nervous system. Wiley Interdiscip Rev Dev Biol. 2013;2:113-29.

\section{Publisher's Note}

Springer Nature remains neutral with regard to jurisdictional claims in published maps and institutional affiliations.

Ready to submit your research? Choose BMC and benefit from:

- fast, convenient online submission

- thorough peer review by experienced researchers in your field

- rapid publication on acceptance

- support for research data, including large and complex data types

- gold Open Access which fosters wider collaboration and increased citations

- maximum visibility for your research: over $100 \mathrm{M}$ website views per year

At $\mathrm{BMC}$, research is always in progress.

Learn more biomedcentral.com/submissions 